\title{
Sunlight exposure and photoprotection behaviour of white Caucasian adolescents in
}

\section{the UK}

M. Gould, ${ }^{1}$ M.D. Farrar, ${ }^{1}$ R. Kift, ${ }^{2}$ J.L. Berry, ${ }^{3}$ M.Z. Mughal, ${ }^{4}$ C. Bundy, ${ }^{1}$ A. Vail ${ }^{5}$, A.R. Webb $^{2}$ and L.E. Rhodes ${ }^{1}$

${ }^{1}$ Centre for Dermatology, Institute of Inflammation and Repair, University of Manchester, Manchester Academic Health Science Centre, Salford Royal NHS Foundation Hospital, Manchester, UK

${ }^{2}$ School of Earth, Atmospheric and Environmental Sciences, University of Manchester, Manchester, UK

${ }^{3}$ Specialist Assay Laboratory (Vitamin D), Institute of Human Development, University of Manchester, Manchester Academic Health Science Centre, Manchester Royal Infirmary, Manchester, UK

${ }^{4}$ Department of Paediatric Endocrinology, Royal Manchester Children's Hospital, Manchester, UK

${ }^{5}$ Institute of Population Health, University of Manchester, Manchester Academic Health Science Centre, Salford Royal NHS Foundation Hospital, Manchester, UK

Running head: Sunlight exposure behaviour of UK adolescents

Correspondence: Lesley E. Rhodes, Photobiology Unit, Dermatology Centre, University of Manchester, Salford Royal NHS Foundation Hospital, Manchester, M6 8HD, UK. E-mail: Lesley.E.Rhodes@manchester.ac.uk; Tel: +44 161 2061150. Fax: +44 1612061156. 
Funding source: This work was supported by a Medical Research Grant from The Bupa Foundation.

The authors declare that there are no conflicts of interest.

Word count: 2706

Figures: 4

Tables: 2

Key Words: Sunlight exposure; photoprotection behaviour; skin type; adolescent.

The definitive version is available at www.blackwell-synergy.com 


\section{Summary}

Background Sun exposure has positive and negative effects on health, yet little is known about the sun-exposure behaviour of UK adolescents, including those more prone or less prone to sunburn.

Objective To examine sun exposure behaviour of UK white Caucasian adolescents including time spent outdoors, holiday behaviour, use of sunscreen and clothing, with assessment for differences between sun-reactive skin type groups.

Methods White Caucasian adolescents (12-15 years) attending schools in Greater Manchester completed a 2 page questionnaire to assess sun exposure and photoprotective behaviour. Results 133 adolescents (median age 13.4 years; 39\% skin type I/II, 61\% skin type III/IV) completed the questionnaire. In summer, adolescents spent significantly longer outdoors at weekends (median $4 \mathrm{~h} /$ day, range $0.25-10)$ than on weekdays $(2,0.25-6 ; P<0.0001)$. When at home in the UK during summer, $44 \%$ reported never wearing sunscreen compared to just $1 \%$ when on a sunny holiday. Sunscreen use was also greater (frequency/coverage) when on a sunny holiday than at home in the UK summer $(P<0.0001)$. Adolescents of skin types I/II (easy burning) spent significantly less time outdoors than skin types III/IV (easy tanning) on summer weekends $(P<0.001)$, summer weekdays $(P<0.05)$ and on a sunny holiday $(P=0.001)$. Furthermore, skin types I/II reported greater sunscreen use during summer in the UK and on sunny holiday (both $P<0.01$ ), and wore clothing covering a greater skin area on a sunny holiday $(P<0.01)$ than skin types III/IV. There was no difference in sun-exposure behaviour/protection between males and females.

Conclusion The greater sun-protective measures reported by adolescents of sun-reactive skin type group I/II than III/IV suggest those who burn more easily are aware of the greater need to protect their skin. However, use of sunscreen during the UK summer is low and may need more effective promotion in adolescents. 


\section{Introduction}

Sunlight exposure in childhood and adolescence is a major risk factor for skin cancer. ${ }^{1,2}$ Skin cancer prevention guidance recommends skin should be protected to avoid sunburn by seeking shade from summer sunlight between $11 \mathrm{am}$ and $3 \mathrm{pm}$, wearing protective clothing and applying sunscreen adequately, especially in people of sun reactive skin types I and II who have higher risk of sunburn and skin cancer. ${ }^{3,4}$

There is little knowledge of the actual sun exposure behaviour of adolescents in the UK, with previous studies tending to focus on older teenagers or younger children. Studies available examining time spent outdoors by adults, adolescents and children in Denmark, the UK and the USA were recently reviewed, with only two involving children and adolescents. ${ }^{5}$ In a UK study, personal UV dosimetry was used to show that adolescents (14-15 years) spent a mean of 1.86 hours outdoors/day on summer weekdays, rising to 2.34 hours/day at weekends. ${ }^{6}$ A Danish cohort of older teenagers (16-19 years) spent an average of 1.18 and 1.72 hours/day outdoors on weekdays and weekends respectively. ${ }^{7}$ There is also a lack of information on attitudes and behaviours relating to photoprotection in adolescents. A questionnaire survey of 288 UK adults on attitudes to and use of sunscreens in northwest England found that $92 \%$ of parents reported their children used sunscreen to some degree, but no information was provided on the age of the children or when sunscreen was worn. ${ }^{8}$ An earlier survey of 2692 UK high school pupils aged $15-16$ years reported $58.1 \%$ of pupils to never wear sunscreen and only $1.3 \%$ to always wear sunscreen on a sunny day at school. ${ }^{9}$

The aim of this study was to address several gaps in our knowledge of sun exposure and photoprotection behaviour of UK white Caucasian adolescents. We performed a questionnaire-based study to examine time spent outdoors, holiday behaviour, sunscreen use, and clothing worn, exploring skin type and gender differences. As an association between first use of sunbeds before the age of 35 and increased risk of melanoma has been reported, ${ }^{10}$ 
and $6 \%$ and $10.8 \%$ respectively of children aged $11-17$ in the UK National Prevalence Study and Six Cities Study reported having used a sunbed, ${ }^{11}$ we also enquired about sunbed use in our questionnaire.

\section{Materials and methods}

\section{Participants}

Subjects were recruited through 5 private (fee-paying) schools and 1 state (free) school in Greater Manchester, UK. Inclusion criteria were: age 12-15 years, male or female, white Caucasian (sun-reactive skin type I-IV ${ }^{12}$ ). Those with a history of a photosensitive disorder were excluded. Written informed consent was obtained from both the children and their parents, and the study adhered to the Declaration of Helsinki principles. Ethical approval was obtained from the Greater Manchester Central Research Ethics Committee (Ref: 10/H1008/58). This study was part of a larger project to examine vitamin D status throughout the year. Questionnaires were completed between January and March 2011.

\section{Skin type assessment}

Subjects were visited at their schools by the researcher, who assessed their sun-reactive skin type using a standardised series of questions relating to history of skin responses to sunlight exposure, modified from Fitzpatrick. ${ }^{12}$

\section{Questionnaire}

Subjects completed a two-page questionnaire, adapted from that used in adults as reported in Stafford et al. ${ }^{13}$ whilst sat at separate desks in a classroom with the researcher available to address any queries. The questionnaire consisted of 10 main questions covering 4 areas: (1) outdoor activities outside of holidays in summer and winter taking account of number 
(before, during and after school) and length of periods outdoors; (2) details of holidays (defined as 3 or more full days away from home) including destination, clothing normally worn during the day and time outdoors; (3) sunscreen use, which comprised frequency of use and area covered, both at home in the UK and when on a sunny holiday; (4) whether the subject had ever used a tanning sunbed and whether they would ever consider using one in the future. Questionnaires were collected by the researcher on completion.

\section{Statistical analysis}

Answers were given a numerical score to enable statistical comparisons between groups (Table 1). The midpoint of each time range was used to calculate hours spent outdoors. Data are presented as median, IQR and range unless otherwise stated. The Mann-Whitney and $\chi^{2}$ tests were used to compare differences between groups and the Wilcoxon signed-rank test was used for within-group comparisons. A value of $\mathrm{p}<0.05$ was considered to indicate a significant difference.

\section{Results}

\section{Subjects}

A total of 133 subjects (53 male; 102 from private, fee-paying schools) were recruited and all completed the questionnaire. Of these, 15 (11\%) were skin type I, 37 (28\%) skin type II, 64 (48\%) skin type III and 17 (13\%) skin type IV. The median age of the group was 13.4 years (range 12.2 to 15.3 ).

\section{Time spent outdoors in the UK}

Nearly all subjects reported spending regular short periods outdoors on weekdays in summer $(132 / 133)$ or winter (129/133) i.e. walking to or from school, at school break/lunchtime, and 
after school. A significantly higher proportion were outdoors during school break/lunchtime and after school in summer than winter $(P<0.0001$; Fig. 1). Subjects spent significantly longer outdoors per day at weekends compared to weekdays in both summer (median $4 \mathrm{~h}$, range $0.25-10$ and $2 \mathrm{~h}, 0.25-6$ respectively; $P<0.0001)$ and winter $(2 \mathrm{~h}, 0.25-8$ and $2 \mathrm{~h}, 0.25-6$ respectively, $P<0.0001$; Fig. 2). The difference between seasons was also significant with subjects spending longer outside on weekends and weekdays respectively in summer compared to winter (both $P<0.0001$ ). There was no difference in time spent outdoors between males and females. Subjects with skin type I/II spent significantly less time outdoors per day than those with skin type III/IV on winter weekends $(P<0.05)$, summer weekends $(P<0.001)$ and summer weekdays $(P<0.05 ;$ Fig. 3$)$. There was no significant difference between the skin type groups for time spent outdoors on winter weekdays.

\section{Time spent outdoors on a sunny holiday}

A total of $108(81 \%)$ subjects had been on at least one holiday in the previous year with 79 $(59 \%)$ in the summer and $35(26 \%)$ in the winter giving a holiday destination with lower latitude, i.e. a sunnier location than the UK. No significant differences were found between holiday locations visited by skin type group I/II compared to III/IV or males compared to females. Subjects spent significantly longer outdoors when on a sunny holiday (median $6 \mathrm{~h}$, range $0.25-10)$ than on summer weekdays $(2 \mathrm{~h}, 0.25-6)$ or weekends $(4 \mathrm{~h}, 0.25-10)$ at home in the UK (both $P<0.0001 ;$ Fig. 2) with those of skin type I/II spending significantly less time outdoors than those of skin type III/IV ( $P=0.001$; Fig 3$)$.

\section{Sunscreen use}

Two (1\%) of the subjects reported never wearing sunscreen. At home in the UK during the summer, 18 (14\%) subjects always wore sunscreen (on all exposed skin or just face and neck) 
and $27(20 \%)$ never wore sunscreen. Sunscreen use was significantly greater $(P<0.0001)$ on a sunny holiday with 73 subjects always wearing sunscreen and only $2(1 \%)$ never wearing sunscreen (Table 2). There was no significant difference in sunscreen use between males and females. However, sunscreen use by subjects with skin type I/II was found to be significantly greater than in those with skin type III/IV, both at home in the UK during the summer $(P<0.01)$ and when on a sunny holiday $(P<0.01)$.

\section{Clothing worn when on a sunny holiday}

When on a sunny holiday, 5 (4\%) subjects reported that they normally wore 'heavyweight clothes covering most of their skin' and $22(17 \%)$ normally wore 'swimsuit/trunks' during the day. The majority of subjects $(85,65 \%)$ normally wore 'shorts and T-shirts'. There was no significant difference in response to the question regarding clothing between male and female subjects. However, subjects with skin type I/II reported wearing clothes that covered a significantly greater area of skin than those of skin type III/IV $(P<0.01$; Fig. 4).

\section{Sunbed use}

Only $2(1 \%)$ subjects ( 1 male, 1 female) stated that they had ever used a sunbed with $5(4 \%)$ subjects ( 2 male, 3 female) stating they thought they would use a sunbed in the future.

\section{School type}

Although comparison of subjects from state and private, fee-paying schools was not an original aim of this study, we found that subjects from the state school spent significantly longer outdoors per day on weekdays than those from private schools in both summer $(P<0.01)$ and winter $(P<0.05)$. There was no significant difference between school types for any other parameter reported above. 


\section{Discussion}

This study explored self-reported sun exposure and photoprotective behaviour in UK white Caucasian adolescents, identifying potential areas to target in future public health campaigns on sun exposure. The two-page questionnaire covering outdoor activities, holidays, clothing, sunscreen use, and also sunbed use, was completed satisfactorily by all subjects, while skin type was assessed face to face by a trained researcher. Key findings were that adolescents spent significantly more time outdoors during times of higher ambient UVR (i.e. summer weekends and on sunny holidays) and sunscreen use was infrequent when at home in the UK during summer, with over $30 \%$ of those surveyed never or rarely using it compared with only $8 \%$ reporting to never/rarely use sunscreen when on a sunny holiday, suggesting public health messages relating to photoprotection in the UK are only partially effective for this group. Nevertheless, adolescents with skin types I and II, more prone to sunburn and skin cancer, were shown to protect themselves from the sun to a significantly greater extent than adolescents with skin types III and IV in several aspects of behaviour.

Adolescents spent significantly longer outdoors in summer than winter for both weekends and weekdays, and also spent more regular short periods outdoors after school, during the school break and at lunchtime in the summer than the winter. This is anticipated, due to longer periods of daylight and warmer, drier weather during the summer period. The finding that longer periods were spent outdoors at weekends than on weekdays in both summer and winter is most likely due to sun-exposure being limited during the week by school attendance, whereas on the weekend there is greater flexibility. An earlier UK study in a narrower age range of adolescents (14-15 years) showed the median time spent outside during the week in spring/summer was 1.65 hours/day, and at the weekend 1.5 hours/day. ${ }^{5,6}$ These lower values than reported in our study ( 2 and 4 hours/day respectively in summer) 
might be influenced by difference in age range and lifestyle, as the majority (77\%) of adolescents in our study attended private fee-paying schools, or could reflect the different methods employed. The questionnaire used in our study relied on recall whereas daily sun exposure diaries were used in the earlier study. Interestingly, a more recent Danish study reported adolescents to spend even less time outdoors ( 0.67 and $0.5 \mathrm{~h} /$ day on weekdays and weekends respectively) as derived from electronic personal UVR dosimetry data. ${ }^{7}$ While the definition of adolescent in this study was older (aged 16-19 years) and only 22 took part, this raises the possibility that adolescents are spending less time outdoors now than in the 1990s.

Time spent outdoors was greater when on sunny holiday compared with summer weekends at home in the UK, with $81 \%$ of adolescents stating that they went away on holiday in the previous year and $82 \%$ of these visiting a sunnier location than the UK. Thus, a significant amount of sun-exposure may be received during such holidays although adolescents do increase their use of sunscreen. Adult studies report higher sunburn rates at home in the UK than when on a sunny holiday away, supporting that the risk of sunburn in the UK is underestimated. ${ }^{8}$ The proportion of adolescents always using sunscreen when at home in the UK summer was $14 \%$. Even fewer (1.3\%) of pupils aged 15-16 years reported always wearing sunscreen in a survey of schoolchildren's behaviour in relation to sunburn although this only considered sunscreen use during the school day. ${ }^{9}$ Low prevalence of sunscreen use in adolescence is a pattern that has been previously reported around the world. ${ }^{14-17}$ Most of these studies were published several years ago and may not represent current trends, particularly in light of national sun awareness campaigns such as the UK's SunSmart that began in $2003 .{ }^{4}$ However, sunscreen use during the UK summer is an area where more targeted guidance for adolescents appears to be required.

Another finding of interest was that adolescents of skin type I/II spent significantly less time outdoors than those with skin type III/IV at all times of the year, other than winter 
weekdays. Thus a habitual pattern of weekend activities may follow those of a particular skin type designation throughout the year. The difference between skin type groups was largest for summer weekends and sunny holidays. Sunscreen use was also found to be greater in skin types I/II both in the UK during the summer and when on a sunny holiday, and this was accompanied by clothing that covered a significantly greater area of skin. This greater sunprotective behaviour by adolescents of skin type I/II may result from this group having past experiences of sunburn, whereas skin types III/IV may place a positive association on sunexposure in view of their predisposition to tan. ${ }^{15}$ Awareness of rising skin cancer incidence in white-skinned people, particularly those of skin type I/II, might also contribute. These findings are consistent with Australian and USA studies examining teenagers and young adults, with those having lighter skin that is more likely to burn practicing greater photoprotection. ${ }^{14-17}$ In the UK, 15-16 year olds described as light-skinned reported a higher incidence of sunburn than those who were dark-skinned, but outdoor and photoprotective behaviours were not assessed. ${ }^{9}$ Thus, differences in these behaviours in adolescents of different skin types in the UK have been clarified in our study.

Earlier studies have reported differences between male and female teenagers' sun exposure and photoprotection behaviour, with females more likely to apply sunscreen and expose greater skin surface area than males. ${ }^{14,16,18}$ Possibly, gender differences in the UK are less apparent due to differing culture and climate, because these were mostly privately educated children or because we examined a younger age group. An encouraging finding in our study was that only $1 \%$ of adolescents had ever used a sunbed, although $4 \%$ said they thought they would in the future. In the UK National Prevalence Study, a similar figure of $1.8 \%$ of 11-14 year olds reported having ever used a sunbed. ${ }^{11}$ Interestingly, that figure increased to $6 \%$ when the age group was expanded to include those aged 15-17 years and was even higher (10.8\%) in 11-17 year olds in the UK Six Cities Study. ${ }^{11}$ 
Limitations of this study include the use of a self-report questionnaire conducted at a single time point. Reliance on recall may have led to inaccuracies in reporting of behaviour that has occurred during the past year. The study group were from relatively privileged backgrounds as the majority of schools involved being fee-paying rather than free (state) schools, and it could be of value to study adolescents of a wider variety of backgrounds. However, this did provide an opportunity to compare behaviour in adolescents from different types of school, although this was not an original study aim. Interestingly, those at state school spent significantly longer outdoors on weekdays (i.e. when attending school) than those at fee-paying schools. This may be due to differences in school curricula and/or extracurricular activities. There was no significant difference between school type for any other parameter. While our population sample cannot be assumed to be representative of UK white Caucasian adolescents in general, the sunbed usage/intention to use revealed similar percentages as reported for 11-14 year olds in the UK National Prevalence Study. ${ }^{11}$

In conclusion, this study has provided novel information on the sun-exposure behaviour of UK adolescents aged 12-15 years, including on sunny holidays and in the UK, frequency and extent of sunscreen use, seasonal behaviour, clothing use and differences between sun reactive skin type groups. Studies of this type are scarce in UK adolescents. Our work suggests that adolescents' sun-exposure behaviour requires more specific targeting by national sun-exposure campaigns. Further research should examine adolescents' attitudes, beliefs and other factors influencing their behaviour, in order that public health guidance is delivered effectively.

\section{Acknowledgements}

We thank The Bupa Foundation for funding. We are grateful to Vivien Robinson for administrative support. 


\section{References}

1. Oliveria SA, Saraiya M, Geller AC, Heneghan MK, Jorgensen C. Sun exposure and risk of melanoma. Arch Dis Child 2006;91:131-8.

2. Kricker A, Armstrong BK, English DR, Heenan PJ. Does intermittent sun exposure cause basal cell carcinoma? A case-control study in Western Australia. Int J Cancer 1995;60:48994.

3. National Institute for Health and Clinical Excellence. Skin cancer prevention: information, resources and environmental changes. 2011. Available at:

http://www.nice.org.uk/guidance/PH32 (last accessed 3rd March 2014).

4. Cancer Research UK. SunSmart Campaign. Available at: http://www.sunsmart.org.uk/ (last accessed 3rd March 2014).

5. Diffey BL. An overview analysis of the time people spend outdoors. Br J Dermatol 2011;164:848-54.

6. Diffey BL, Gibson CJ, Haylock R, McKinlay AF. Outdoor ultraviolet exposure of children and adolescents. Br J Dermatol 1996;134:1030-4.

7. Thieden E, Philipsen P, Heydenreich J, Wulf H. UV radiation exposure related to age, sex, occupation, and sun behavior based on time-stamped personal dosimeter readings. Arch Dermatol 2004;140:197-203.

8. Ling TC, Faulkner C, Rhodes LE. A questionnaire survey of attitudes to and usage of sunscreens in northwest England. Photodermatol Photoimmunol Photomed 2003;19:98-101. 9. Horsley L, Charlton A, Waterman C. Current action for skin cancer risk reduction in English schools: pupils' behaviour in relation to sunburn. Health Educ Res 2002;17:715-31. 10. International Agency for Research on Cancer. Exposure to artificial UV radiation and skin cancer. IARC Working Group Report. Vol. 1. Lyon, France: IARC, 2006. 
11. Thomson CS, Woolnough S, Wickenden M, Hiom S, Twelves CJ. Sunbed use in children aged 11-17 in England: face to face quota sampling surveys in the National Prevalence Study and Six Cities Study. BMJ 2010;340:c877.

12. Fitzpatrick TB. The validity and practicality of sun-reactive skin types I through VI. Arch Dermatol 1988;124: 869-71.

13. Stafford R, Farrar MD, Kift R et al. The impact of photosensitivity disorders on aspects of lifestyle. Br J Dermatol 2010;163:817-22.

14. Broadstock M, Borland R, Hill D. Knowledge, attitudes and reported behaviours relevant to sun protection and suntanning in adolescents. Psychol Health 1996;11:527-39.

15. Clarke VA, Williams T, Arthey S. Skin type and optimistic bias in relation to the sun protection and suntanning behaviors of young adults. J Behav Med 1997;20:207-22.

16. Geller AC, Colditz G, Oliveria S et al. Use of sunscreen, sunburning rates, and tanning bed use among more than 10,000 US children and adolescents. Pediatrics 2002;109:1009-14. 17. Livingston P, White V, Hayman J, Dobbinson S. Australian adolescents' sun protection behavior: who are we kidding? Prev Med 2007;44:508-12.

18. Banks BA, Silverman RA, Schwartz RH, Tunnessen WW. Attitudes of teenagers toward sun exposure and sunscreen use. Pediatrics 1992;89:40-2. 
Table 1 Scoring system used for statistical analyses of time outdoors, sunscreen use and clothing.

\begin{tabular}{ll}
$\begin{array}{l}\text { Topic area and available responses } \\
\text { Hours outdoors }\end{array}$ & Score \\
\hline 30 min or less & 1 \\
\hline$>30$ min to $<1 \mathrm{~h}$ & 2 \\
\hline $1 \mathrm{~h}$ to $<3 \mathrm{~h}$ & 3 \\
\hline $3 \mathrm{~h}$ to $<5 \mathrm{~h}$ & 4 \\
\hline $5 \mathrm{~h}$ to $<7 \mathrm{~h}$ & 5 \\
\hline $7 \mathrm{~h}$ to $<9 \mathrm{~h}$ & 6 \\
\hline $9 \mathrm{~h}$ or $\mathrm{more}$ & 7
\end{tabular}

Use of sunscreen

\begin{tabular}{ll}
\hline Always, all exposed skin & 1 \\
\hline Always, face and neck & 2 \\
\hline Usually, all exposed skin & 3 \\
\hline Usually, face and neck & 4 \\
\hline Sometimes, all exposed skin & 5 \\
\hline Sometimes, face and neck & 6 \\
\hline Rarely, all exposed skin & 7 \\
\hline Rarely, face and neck & 8 \\
\hline Never & 9 \\
\hline
\end{tabular}

Clothing

\begin{tabular}{ll}
\hline Full body cover & 1 \\
\hline Heavyweight clothes covering most of the skin & 2 \\
\hline Lightweight clothes & 3 \\
\hline Shorts and T-shirt & 4 \\
\hline Bathing suit/bikini & 5 \\
\hline
\end{tabular}


Table 2 Responses to the question 'How often do you use sunscreen?'

\begin{tabular}{lcc} 
& & \multicolumn{2}{c}{ Number of subjects (\%) } \\
& At home during the summer & On a sunny holiday \\
Always, all exposed skin & $13(10)$ & $68(51)$ \\
\hline Always, face and neck & $5(4)$ & $5(4)$ \\
\hline Usually, all exposed skin & $12(9)$ & $28(21)$ \\
\hline Usually, face and neck & $11(8)$ & $9(6)$ \\
\hline $\begin{array}{l}\text { Sometimes, all exposed } \\
\text { skin }\end{array}$ & $22(17)$ & $5(4)$ \\
\hline Sometimes, face and neck & $12(9)$ & $5(4)$ \\
\hline Rarely, all exposed skin & $16(12)$ & $3(2)$ \\
\hline Rarely, face and neck & $15(11)$ & $2(1)$ \\
\hline Never & $27(20)$ & 9 \\
\hline
\end{tabular}




\section{Figure Legends}

Figure 1 Number of subjects spending regular short periods outdoors during the week in both summer and winter. Significant differences and $P$-values are indicated.

Figure 2 Time spent outdoors on weekdays and weekends during summer and winter at home in the UK, and when on a sunny holiday. Plot shows range (whiskers), lower and upper quartiles (boxes) and median (thick lines) of number of hours spent outdoors per day. Significant differences and $P$-values are described in the text.

Figure 3 Time spent outdoors by subjects of skin type I/ II and III/IV on weekdays and weekends during summer and winter at home in the UK, and when on a sunny holiday. Plot shows range (whiskers), lower and upper quartiles (boxes) and the median (thick lines) of number of hours spent outdoors per day. Significant differences and $P$-values are indicated.

Figure 4 Normal clothing worn during the day by subjects of skin type I/II and III/IV when on a sunny holiday. 
Figure 1.

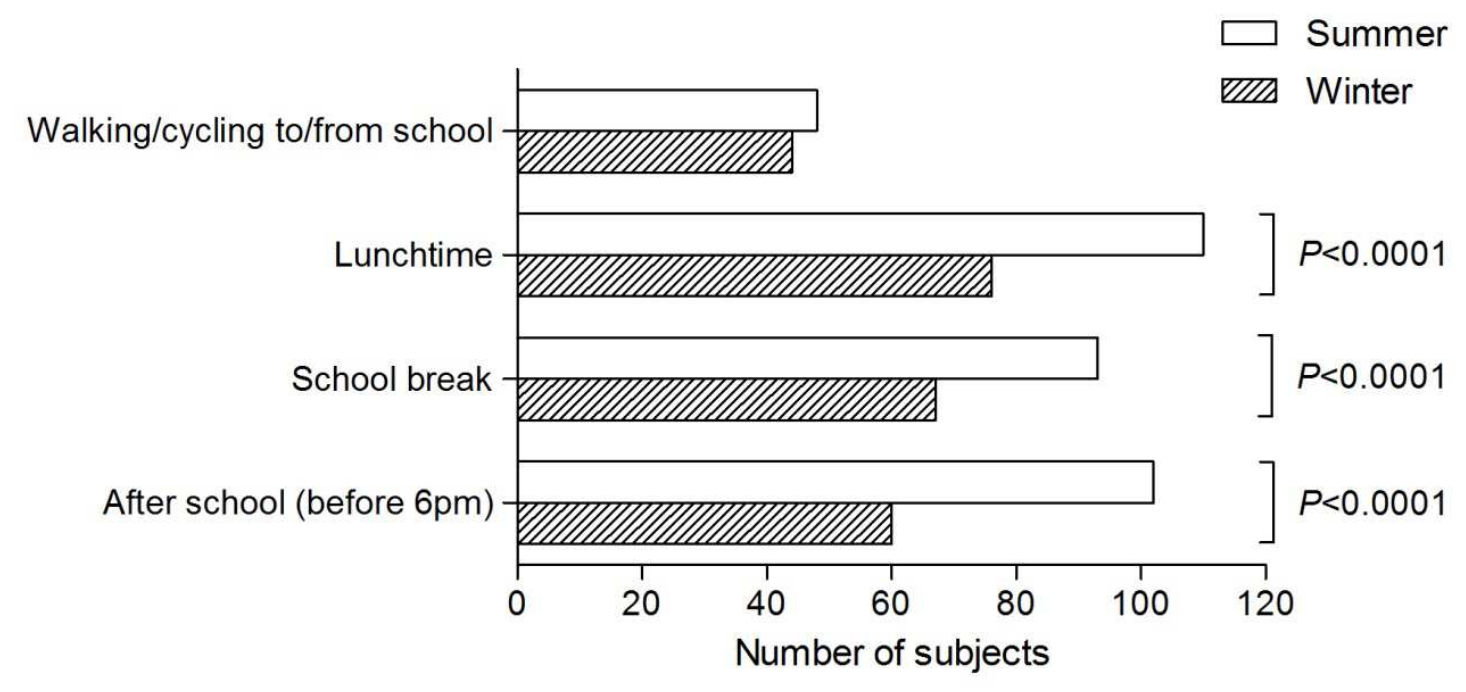


Figure 2.

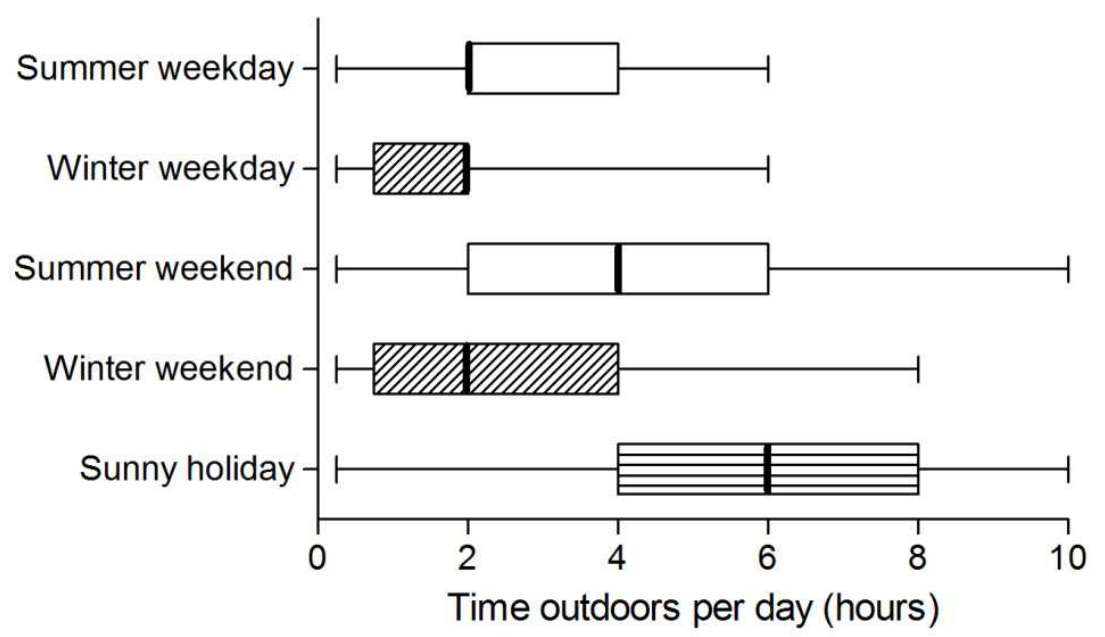


Figure 3.

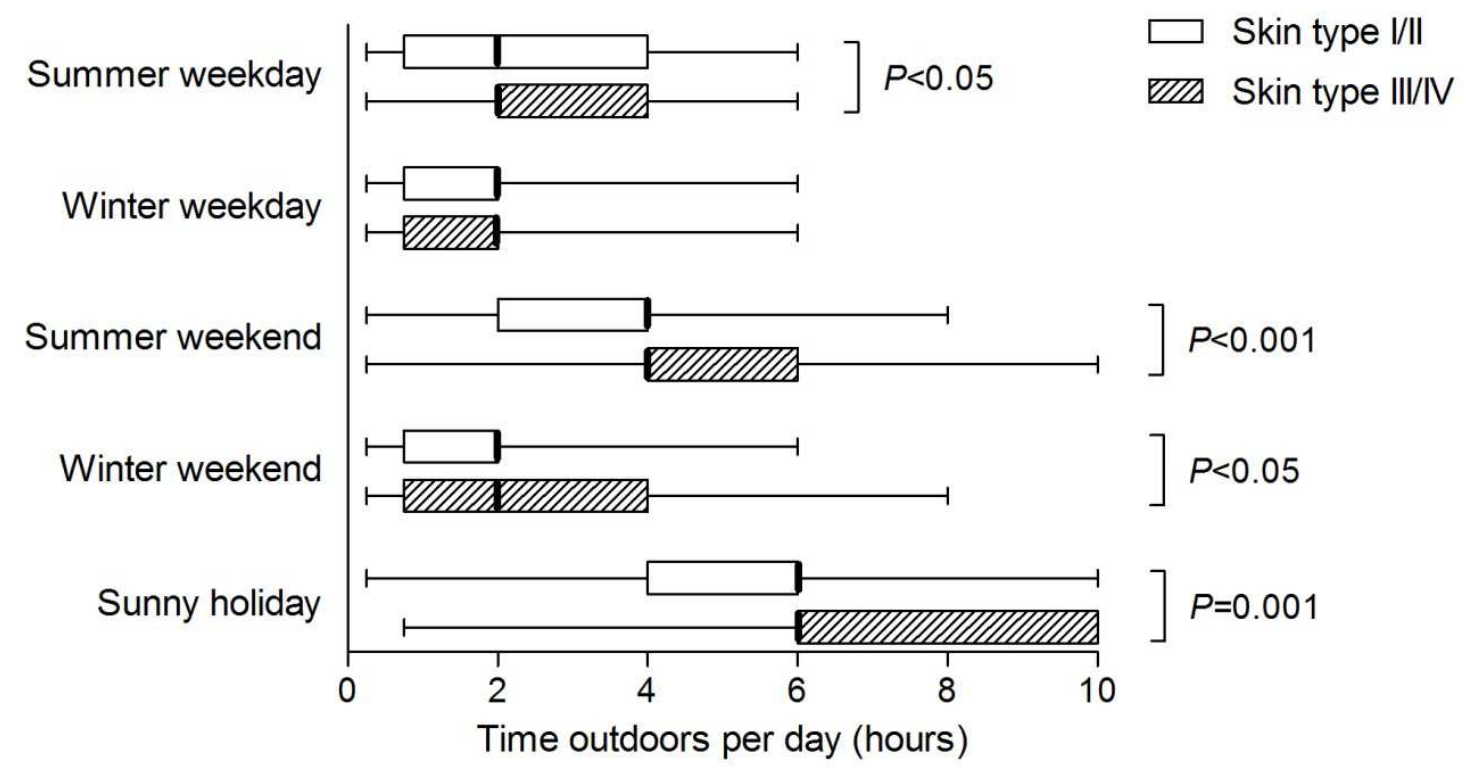


Figure 4.

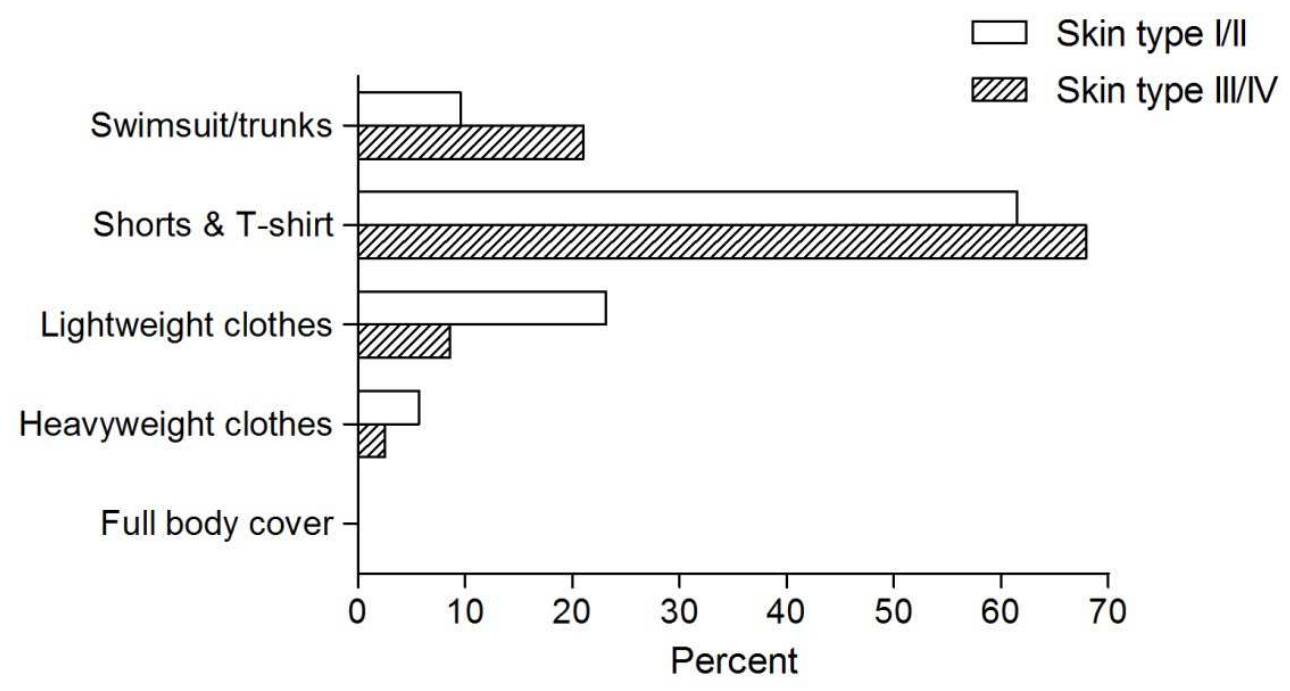

